(1)

CrossMark

\title{
The disease model: implications for clinical practice
}

\author{
Alvar Agusti ${ }^{1,2}$ \\ Affiliations: ${ }^{1}$ Respiratory Institute, Hospital Clinic, Institut d'investigacions Biomèdiques August Pi i Sunyer \\ (IDIBAPS), University of Barcelona, Barcelona, Spain. ${ }^{2}$ Centro de Investigación Biomédica En Red
} Enfermedades Respiratorias (CIBERES), Spain.

Correspondence: Alvar Agusti, Respiratory Institute, Hospital Clinic, Villarroel 170, 08036 Barcelona, Spain. E-mail: AAGUSTIAclinic.cat

@ERSpublications

Chronic noncommunicable diseases challenge the traditional "disease model" of acute diseases and it is proposed here that their clinical management requires a different "disease model" based on the presence of so-called treatable traits http://ow.ly/9Z1930iYZX3

Cite this article as: Agusti A. The disease model: implications for clinical practice. Eur Respir J 2018; 51: 1800188 [https://doi.org/10.1183/13993003.00188-2018].

Since the most remote origins of medicine, thousands of years ago, clinical practice has been based on the so-called "disease model" [1]. As shown in figure 1a, this model establishes that a number of risk factors and causative triggers interact to produce a "disease" characterised by specific pathology that manifests by a series of symptoms and signs which, importantly, guide the diagnosis and treatment of the "disease". More recently, this paradigm has been enriched by the use of complementary diagnostic techniques that are used to confirm (or refute) the clinical diagnosis (as well as the proposed treatment).

This simple "disease model" generally works well for acute diseases in otherwise healthy subjects because they most often involve a single organ system and have a well-defined and short clinical course (e.g. trauma, infections and many others). However, this is not the case when we consider the clinical management of chronic, noncommunicable diseases which, today, are far more prevalent than acute diseases [1]. In fact, chronic noncommunicable diseases have been identified by the General Assembly of the United Nations as the main public health problem we face at the beginning of the 21st century [2]. Furthermore, somewhat ironically, this is in part due to the success we have had managing acute diseases. For instance, mortality due to coronary artery disease has decreased significantly over the last decades [3]. This has led to a clear increase in life expectancy that opens the possibility of suffering other chronic diseases, such as chronic obstructive pulmonary disease [4].

The "disease model" of chronic noncommunicable diseases (figure 1b) is far more complex than that of acute diseases (figure 1a). It includes more risk factors and triggers that often interact (e.g. ageing and smoking) to damage several organ systems (e.g. cardiovascular and respiratory) simultaneously. As a result, the symptoms perceived by the patient may have a multi-site origin. This makes a precise diagnosis and a specific treatment difficult and, as a result, these patients often end up receiving multiple therapies at the same time that can also interact [1]. Finally, it is also of note that the clinical management of chronic noncommunicable diseases has to consider nonbiological aspects, such patient preferences and family and social support, which often determine compliance with therapy and directly influence the clinical outcome [1]. All in all, the original and simple "disease model" (figure 1a) probably needs to be abandoned when managing chronic noncommunicable diseases. The question is then, what is the alternative model, if any? 
a) Acute diseases (short, on/off course, single organ)

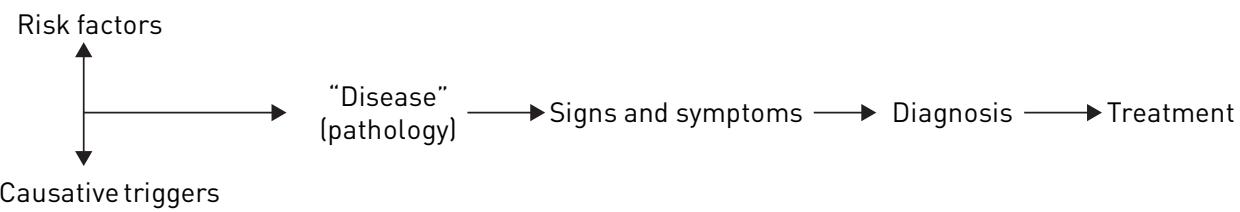

b) Chronic diseases (long, undefined course, multiple organs\#)

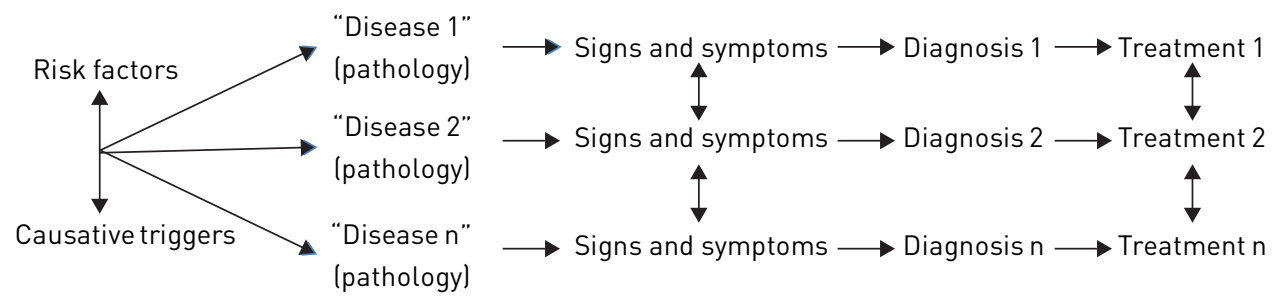

FIGURE 1 Pictorial representation of a) the traditional disease model, which is appropriate for acute diseases, and b) a more complex model that describes more realistically what happens in chronic noncommunicable diseases. \#: nonbiological factors (social, familial, life-style and others) can play a significant role in what most often matters to patients: symptoms.

One of the anonymous reviewers of this article argues that we may not need a disease model at all because artificial intelligence (AI) will revolutionise medicine in such a way that it will never be the same any more (medicine 3.0) [5]. Although I agree that this is certainly a possibility, I also believe that in the meantime we still need some kind of "disease model" to work with. In this context, a potential alternative model for the clinical management of chronic noncommunicable diseases may be based on the so-called "treatable traits" [6-8], which explicitly avoids the old, restrictive, Oslerian diagnostic labels that accompany the traditional "disease model" [9]. A treatable trait can be identified on the basis of phenotypic (clinical) recognition or through a deeper understanding of the causal pathways (endotypes) via validated biomarkers $[6,7,10]$. Importantly, treatable traits are not mutually exclusive (i.e. can coexist in the same patient) and can change with time (spontaneously or as a result of treatment) $[6,7,10]$. Of note, too, as appropriately suggested by the same reviewer, it would be of great relevance to recognise those treatable traits that we cannot prevent or treat yet ("untreatable traits") because they then become a goal for biomedical research.

The current availability of electronic health records should easily allow us to build a "control panel" (similar to those that pilots use in aeroplane cabins to fly safely and effectively) that presents the treatable traits present in any given individual to the practising clinician in a user-friendly manner [11]. Furthermore, current computing power and AI strategies [5] can conspire to curate the current guidelines plethora [1] and present the practising clinician with a precision medicine strategy that provides the best therapeutic options to the specific patient she/he is taking care of, based on both guideline recommendations and specific needs of the patient. Needless to say, this proposal requires formal prospective validation in appropriately designed clinical trials. Yet, it offers a potential alternative to implement a new "disease model" for chronic noncommunicable diseases. In fact, as suggested by another reviewer, good clinical practice in multimorbid patients is already based on treatable traits because there is no alternative way to approach these patients and, indeed, an inter-societal working group, involving the European Respiratory Society and other stakeholders (particularly the internal medicine community), may be needed to change textbooks, curricula and organisation of care in order to translate this message to clinical practice.

Acknowledgements: I would like to sincerely thank the two anonymous reviewers of this article for their very helpful comments and suggestions, which are now included in the text and have, in my mind, contributed significantly to enrich it.

Conflict of interest: A. Agusti reports grants from GSK, personal fees from Novartis and Chiesi (for lectures), grants and personal fees from Astra-Zeneca and personal fees from Boheringer-Ingelheim (for lectures and advisory boards), outside the submitted work.

\section{References}

1 Tinetti ME, Fried T. The end of the disease era. Am J Med 2004; 116: 179-185. 
2 Rosenbaum L, Lamas D. Facing a "slow-motion disaster" - the UN meeting on noncommunicable diseases. N Engl J Med 2011; 365: 2345-2348.

3 Rosamond WD, Chambless LE, Folsom AR, et al. Trends in the incidence of myocardial infarction and in mortality due to coronary heart disease, 1987 to 1994. N Engl J Med 1998; 339: 861-867.

4 Vogelmeier CF, Criner GJ, Martinez FJ, et al. Global Strategy for the Diagnosis, Management, and Prevention of Chronic Obstructive Lung Disease 2017 Report: GOLD Executive Summary. Eur Respir J 2017; 49: 1700214.

$5 \quad$ The Lancet. Artificial intelligence in health care: within touching distance. Lancet 2017; 390: 2739.

6 Agusti A, Bel E, Thomas M, et al. Treatable traits: toward precision medicine of chronic airway diseases. Eur Respir J 2016; 47: 410-419.

7 Agustí A, Bafadhel M, Beasley $\mathrm{R}$, et al. Precision medicine in airway diseases: moving to clinical practice. Eur Respir J 2017; 50: 1701655.

8 Pavord ID, Beasley R, Agusti A, et al. After asthma: redefining airways diseases. Lancet 2018; 391: 350-400.

9 Vanfleteren LE, Kocks JW, Stone IS, et al. Moving from the Oslerian paradigm to the post-genomic era: are asthma and COPD outdated terms? Thorax 2014; 69: 72-79.

10 Agusti A, Celli B, Faner R. What does endotyping mean for treatment in chronic obstructive pulmonary disease? Lancet 2017; 390: 980-987.

11 Agusti A, MacNee W. The COPD control panel: towards personalised medicine in COPD. Thorax 2013; 68: $687-690$. 